\title{
HAE: Enzymsubstitution bleibt Therapie der Wahl
}

— In Deutschland sind nur schätzungsweise 1.500 Personen von einem hereditären Angioödem (HAE) betroffen - einer der Gründe, warum diese tückische Erkrankung noch immer häufig lange Zeit als Quincke-Ödem fehlgedeutet wird. Ursache der autosomal vererbten Erkrankung ist ein Mangel oder eine funktionelle Störung des C1-Esterase-Inhibitors (C1-INH), das an der Regulation des Komplementsystems beteiligt ist.

Typische Symptome sind wiederkehrende Schwellungen an Händen, Füßen oder im Gesicht, aber auch an den Schleimhäuten, wie Dr. Emel Aygören-Pürsün, Frankfurt, berichtete. Sind die Schleimhäute im Bereich des Kehlkopfs betroffen, kann eine
Attacke sogar lebensbedrohlich sein, eine Schwellung der Darmschleimhaut kann zum Darmverschluss führen. Häufig kommt es auch zu krampfartigen Bauchattacken. Die Ödeme bilden sich in der Regel innerhalb von einem bis acht Tagen zurück, kommen jedoch häufig wieder. Manche Patienten sind zwei- bis dreimal pro Woche betroffen, so Aygören-Pürsün. Bei Verdacht auf HAE muss die Diagnose per Bluttest gesichert werden.

An der HAE-Ambulanz in Frankfurt, der weltweit größten ihrer Art, werden inzwischen mehr als 500 HAE-Patienten behandelt. Therapie der Wahl im akuten Anfall ist hier die i.v. Injektion eines C1-INH-Konzentrats (Berinert ${ }^{\circledR}$ ), die geschulte Patienten selbst vornehmen können. Die Substitutionstherapie sollte individuell erfolgen, da die Attackenfrequenz stark variiert, betonte Dr. Inmaculada Martinez-Saguer. Spüren die Patienten erste Symptome, sollten sie sich das Präparat injizieren um die Attacke zu kupieren.

Diese Therapie sei gut verträglich, betonte die Ärztin, während die Behandlungsalternative in Form oraler Androgen-Derivate insbesondere bei Frauen oft Probleme aufwerfe.

Der seit kurzem verfügbare BradykininRezeptorantagonist Icatibant stellt eine weitere subkutane Therapieoption dar, die nach Ansicht der Frankfurter Experten allerdings eher für Patienten mit selteneren Attacken geeignet ist.

Pressegespräch zur HAE-Ambulanz, Frankfurt, 21.1.2010 (Veranstalter: CSL Behring)

\section{SIT: Bei der Präparatewahl auf Evidenz achten}

- Präparate für die spezifische Immuntherapie (SIT) mit häufig verwendeten Allergenen wie Süßgräsern, Frühblühern oder Milben müssen nach der Therapieallergene-Verordnung ab Ende 2011 - anders als jetzt - alle eine Zulassung haben. Daher rät Prof. Claus Bachert, Krefeld/Gent, sich über die Evidenz der Produkte zu informieren. Denn nach Angaben von PD Dr. Randolf Brehler, Münster, gibt es bereits heute einige, für die qualitativ hochwertige Studien vorliegen.

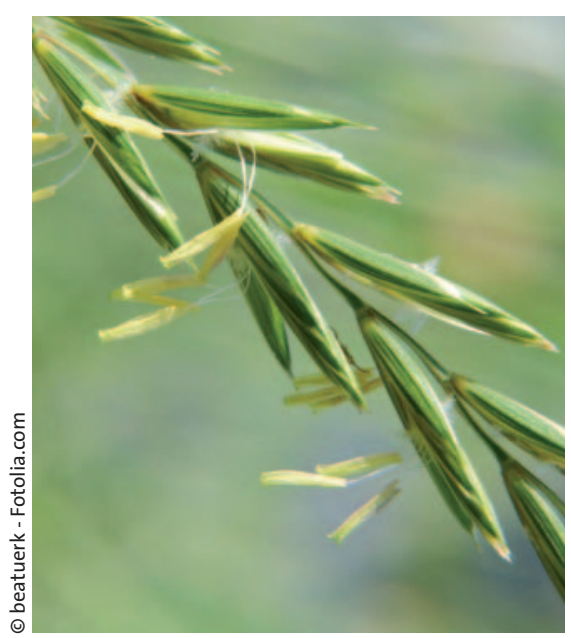

Im April und Mai beginnt sie wieder: die Hochsaison der Gräserpollen.
Zu ihnen gehört Oralair ${ }^{\circledR}$, ein Extrakt aus fünf Gräserpollen, zu dem Studien mit mehr als 2.500 Patienten laufen bzw. bereits abgeschlossen sind. Die Sublingualtablette konnte laut Brehler in einer Dosis von 300 IR bei Erwachsenen mit Gräserpollenallergie gegenüber Placebo die Symptome innerhalb des ersten Jahres um $34 \%$ und nach drei Jahren um $50 \%$ (je $p<0,0001$ ) reduzieren. Zudem besserte sie die Lebensqualität während einer Saison signifikant ( $p<0,0001$; Horak, et al. Clin Exp Allergy 2009; 39: 394-400). Bei Kindern und Jugendlichen reduzierte sie die Symptome innerhalb einer Saison gegenüber Placebo um 39\% ( $p<0,001$; Wahn et al. Allergy Clin Immunol 2009; 123: 160-6).

Nach den Ausführungen Prof. Dr. KarlChristian Bergmann, Berlin, belegte zudem erstmals eine Phase-II/III-Studie die Wirksamkeit einer Sublingualtablette bei Erwachsenen mit Milbenallergie, deren Zulassung in Vorbereitung ist: Sie besserte in einer Dosis von 300 IR innerhalb von einem Jahr den Symptom-Score $(p=0,0136)$ und die Lebensqualität $(p=0,0191)$ gegenüber Placebo signifikant.

Expert-Meeting „Die neue Dimension der evidenzbasierten SIT“, Berlin, 20.1.2010 (Veranstalter: Stallergenes)

\section{Gereizte Haut: Was Patienten nutzt}

- Dass Dexpanthenol (z.B. in Bepanthen ${ }^{\circledR}$ Wund- und Heilsalbe) zur Behandlung gereizter Hautareale geeignet ist, wurde bereits durch eine Vielzahl von Untersuchungen belegt. Nun hat sich erstmals eine Studie unter Leitung von Prof. Matthias Augustin am Kompetenzzentrum für Versorgungsforschung in der Dermtatologei (CVderm) mit dem patientenrelevanten Nutzen dieser Behandlung beschäftigt. Das Ergebnis: Von den 1.886 Patienten, die im Rahmen der Selbstmedikation Bepanthen ${ }^{\circledR}$ Wund- und Heilsalbe verwendeten, berichteten 94,7\% über einen Behandlungserfolg. Symptome wie Trockenheit, Erythem und Schuppung besserten sich signifikant. 91,6\% der Patienten waren mit der Behandlung zufrieden oder sehr zufrieden; 96,5\% würden sie erneut anwenden und 96,1\% sie an andere weiterempfehlen.

dka

Nach Informationen von Bayer HealthCare 